\title{
FACIAL TRACKING WITH HEAD POSE ESTIMATION IN STEREO VISION
}

\author{
Yu Huang, Tom Huang \\ Beckman Institute, UIUC, Urbana, Illinois, US, IL61801 \\ e-mail: \{yuhuang, huang \} @ifp.uiuc.edu
}

\begin{abstract}
Facial tracking is a key step for face modeling, coding and animation. In this paper we present a facial tracking method in stereo vision. We use an efficient representation of 3-D face motion, consisting of global head motion and local facial deformation. Stereo vision is utilized to measure 3-D coordinates of the face, the global and local motion parameters of the tracked face is solved respectively. From the 3-D space to the left and right image space we employ re-projection to realize 3-D tracking. Experiment results of our method are given to demonstrate its performance.
\end{abstract}

Key words: Facial tracking, stereo vision, head pose

\section{INTRODUCTION}

Because of many potentially important applications, "Looking at People" is currently one of the most active application domains in computer vision. Among them, there is a vast body of work on human facial tracking (including the eyes, nose and mouth), with applications such as face recognition, facial expression analysis, face modeling, coding and animation [1-15].

Faces are highly dynamic patterns those undergo many non-rigid transformations. The previous methods of facial tracking can be classified as 2-D or 3-D. 2-D trackers can be based on deformable or rigid patches, or on edge or feature detectors $[3,14,18,13,9,12]$, but 3 -D properties of the face will handle large variations of face orientations. One method is to build a physically-based model that describes the muscular structure of the face $[5,17,16,1,7]$. Another method employs an analysis-by-synthesis mechanism where the face images are analyzed by comparing them to synthetic face images $[4,6,8,10,15]$.

In this paper, we propose a stereo facial tracking method, in which 3-D information is fully utilized to estimate the pose of the head and the expression parameters. We give an efficient representation of the facial motion describing the main expression motion and head motion. From the 3-D space to the left and right image space we employ re-projection to realize stereo tracking. The tracking result can be used for facial animation and coding.

\subsection{Related Work}

They are few works about facial stereo tracking.

[13] Matsumoto et.al construct a real-time stereo vision system for facial tracking and head pose estimation. They pick six fiducial points at the corners of the mouth and the eyes as the tracked features, where the 2-D tracking is based on 2-D template matching (temporal and stereo). These 2-D coordinates are employed to reconstruct the 3$\mathrm{D}$ points those can be used to estimate the head pose in a weighted LS framework. Afterwards they adjust the 3-D points' coordinates by the estimated motion parameters (rotation and translation), then re-project the results into 2D image planes (both left and right) those are the new 2-D positions of the tracked features. Here they assume these features do not perform obvious non-rigid motion such as facial expression.

[8] Gokturk et.al recently use the analysis-by-synthesis mechanism for facial tracking. They train the facial expression of a front-view face in 3-D space, where a stereo vision tracking system are used for obtain the 3-D coordinates of the features in the eyebrows, eyes, nose and mouth. After 3-D expression learning, they will use the result to help monocular facial tracking with head motion. The 2-D feature tracking is based on the KLT tracker [18].

\section{FRAMEWORK OF FACIAL TRACKING}

Our Algorithm procedure of stereo facial tracking is:

1) Spatio-temporal matching in stereo image to find the 2-D position in the left camera at the next time instant;

2) Spatio-temporal matching in stereo images to find the 2-D position in the right camera at the next time instant;

3) 3-D reconstruction using the matched points to get the 3-D coordinates;

4) Calculation of global (the head's) motion between two time instants;

5) Calculation of local motion (the expression);

6) Re-adjustment of 3-D coordinates (from the head pose and expression);

7) $2 \mathrm{D}$ projection of the 3-D points to update the search area for template matching;

8) Go 1) - 7) at the next time instant.

\subsection{Representation of Facial Motion}


Deformable models are parameterized shapes that deform due to forces according to physical laws. In Figure 1 we illustrate our model: we select some points on the facial features: 1 point between the eyes, 3 points of each eyebrow, 2 corners of each eye, 2 points of each eyelid, 2 nostrils, the tip of the nose, 2 corners and another 6 points of the mouth. Here we can make simple triangulation in the middle face area. The points of eyes and the nose are assumed to be independent from facial expression, so they are used to estimate the head motion. The points of eyebrows and the mouth would be employed to estimate the facial mimic after the head motion compensation. An example of our model fitted to a real image is shown too.

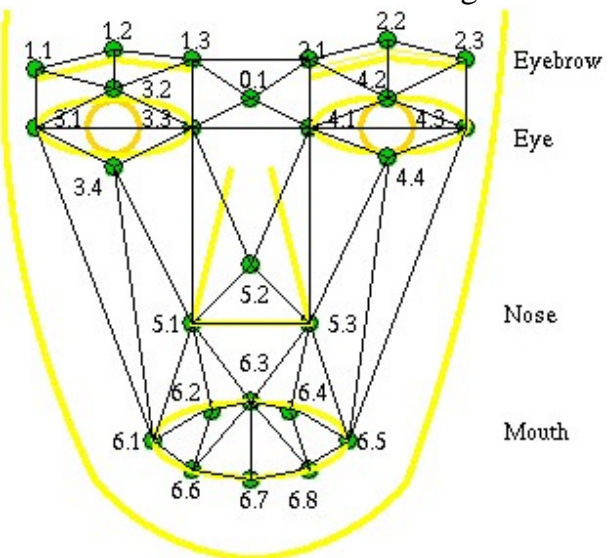

(a) Face Model

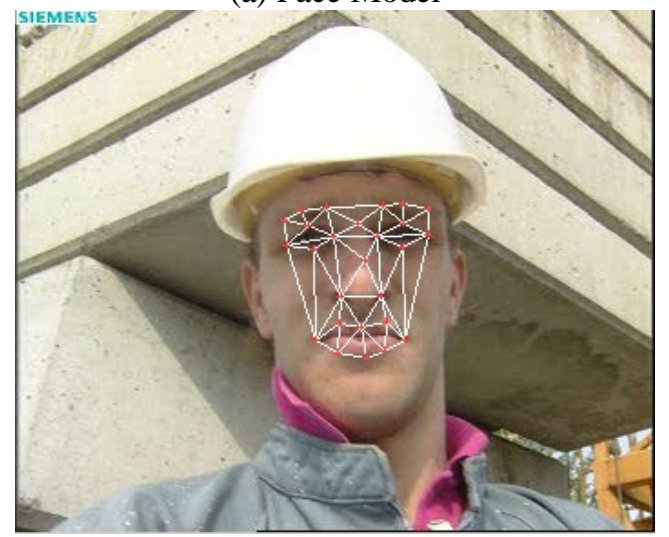

(b) Model Fitting

Figure 13 -D Face Model and its Fitting on the Image

\subsubsection{Rigid head motion}

It is reasonable to assume the head is a rigid object, so

$$
p=R p_{0}+T,
$$

With $p=(x, y, z)^{T}$ as the 3-D feature points on the head, $p_{0}=\left(x_{0}, y_{0}, z_{0}\right)^{T}$ is the corresponding points in the initial position, $R, T$ as the orientation matrix and the translation vector of the head relative to the initial pose.

\subsubsection{Non-rigid expression motion}

The selection of an adequate family of shape functions is dictated by accuracy and compactness requirements. The most frequent choice consists of the second order interpolating polynomials, especially parabolic arcs.
Nevertheless, quadric representations prove to be too rigid for accurately dealing with a wide variety of facial expressions, in particular those involving large deformations of the mouth. Known as versatile modeling tools in computer graphics, spline-based representations have been successfully used for facial component modeling [7,17]. Harmonics-based modeling has been shown to provide more accurate results [11], but does not allow an intuitive geometric manipulation of the deformed template.

Here we span the 3-D displacement space by the following orthogonal bases

$$
\vec{D}\left(p_{0}\right)=\sum_{i=1}^{l} \sum_{j=1}^{m} \sum_{k=1}^{n} E_{i j k} \vec{\alpha}_{i j k},
$$

Where $\vec{\alpha}_{i j k}=\left(\alpha_{i j k}^{x}, \alpha_{i j k}^{y}, \alpha_{i j k}^{z}\right)^{T}$ is the vector of facial expression parameters, and $E_{i j k}=\left(\vec{e}_{i j k}^{x}, \vec{e}_{i j k}^{y}, \vec{e}_{i j k}^{z}\right)^{T}$ is the matrix of the span space by the following basis functions,

$$
\begin{aligned}
& \vec{e}_{i j k}^{x}=\left(2 \sin \left(i \pi x_{g}\right) \cos \left(j \pi y_{g}\right) \cos \left(k \pi z_{g}\right) / C_{i j k}, 0,0\right)^{T}, \\
& \vec{e}_{i j k}^{y}=\left(0,2 \cos \left(i \pi x_{g}\right) \sin \left(j \pi y_{g}\right) \cos \left(k \pi z_{g}\right) / C_{i j k}, 0\right)^{T}, \\
& \vec{e}_{i j k}^{z}=\left(0,0,2 \cos \left(i \pi x_{g}\right) \cos \left(j \pi y_{g}\right) \sin \left(k \pi z_{g}\right) / C_{i j k}\right)^{T},
\end{aligned}
$$

Where $C_{i j k}=K \pi^{2}\left(i^{2}+j^{2}+k^{2}\right)$ are the normalization constants and $K$ is a scale factor, $p_{g}=\left(x_{g}, y_{g}, z_{g}\right)^{T}$ is the normalized point enclosed by the unit cube. In our implementation, $1, \mathrm{~m}, \mathrm{n}$ are set to be 2 . So the number of expression parameters is $2 \times 2 \times 2 \times 3=24$.

In the initialization we get a standard front face without expression (a neutral face), and measure $p_{b},\left(s_{x}, s_{y}, s_{z}\right)$ according to some proportion relationships of geometrical measuring occurred on the human head model.

\subsubsection{Combined motion}

Now the whole motion is combined by global and local motion as

$$
p=R\left(p_{0}+\vec{D}\left(p_{0}\right)\right)+T .
$$

Our face motion model allows the deformation of the face independent from the orientation of the head. It can guarantee the unique deformation parameters to describe the facial expression.

\subsection{Solution of the Face Pose and Expression}

We adopt the idea of virtual springs to solve the motion estimation problem in an iterative framework. Here we assume the motion is smooth both in global head movement and local facial deformation. The weight corresponds to the stiffness of the springs, and the object function corresponds to the elastic energy of the springs.

\subsubsection{Rigid head motion}

Here only eight points at corners of eyes and the nose are used, they are assumed not changed in the expression process.

Head pose can be estimated as follows 


$$
\min F_{0}=\sum_{i} w_{i}\left(p_{i}-R p_{i 0}-T\right)^{T}\left(p_{i}-R p_{i 0}-T\right),
$$

Here it can be solved by SVD according to [2].

\subsubsection{Non-rigid expression motion}

After being compensated by the head motion, the left eighteen points at eyebrows, eyelids and the mouth will be used to calculate the expression parameters. We can imagine the head has been "adjusted" to the front view now:

Let $p_{0}^{\prime}=R^{T}(p-T)$, then $d p_{0}^{\prime}=\left(p_{0}^{\prime}-p_{0}\right)=\vec{D}\left(p_{0}\right)$, the goal is changed to solve the following WLS problem,

$$
\min F^{\prime \prime}=\sum_{i} w_{i}\left(d p^{\prime}{ }_{0 i}-E_{i} \xi\right)^{T}\left(d p^{\prime}{ }_{0 i}-E_{i} \xi\right),
$$

Where $\xi$ is the vector of 24 facial expression parameters, $E_{i}$ is the matrix of the deformation span bases. The weights $w_{i}$ are the same to the global motion.

The LS solution is given by

$$
\xi=\left[\sum_{i}\left(w_{i} E_{i}^{T} E_{i}\right)\right]^{-1}\left[\sum_{i} w_{i} E_{i}^{T} d p^{\prime}{ }_{0 i}\right] .
$$

\subsection{Reconstruction of 3-D Coordinates}

We calibrate each camera (left and right) with Zhang's flexible method [20]: A 3D point is denoted as $p=\left[\begin{array}{lll}x & y & z\end{array}\right]^{T}$, its $2 \mathrm{D}$ image points in a stereo vision system are $P_{l}=\left[u_{l}, v_{l}\right]^{T}$ and $P_{r}=\left[u_{r}, v_{r}\right]^{T}$, so the relationship between the $3 \mathrm{D}$ point and its image projections in left and right camera is given by

$$
s_{j}\left[\begin{array}{c}
P_{j} \\
1
\end{array}\right]=A_{j}\left[\begin{array}{ll}
R_{j} & T_{j}
\end{array}\right]\left[\begin{array}{l}
p \\
1
\end{array}\right],(j=l, r),
$$

Where $s_{j}$ are scale factors, $\left(R_{j} T_{j}\right)$ are extrinsic parameters, and $A_{j}$ is the intrinsic matrix given by

$$
A_{j}=\left[\begin{array}{ccc}
\alpha_{j} & \gamma_{j} & u_{j 0} \\
0 & \beta_{j} & v_{j 0} \\
0 & 0 & 1
\end{array}\right],(j=l, r),
$$

With $\left(u_{j 0}, v_{j 0}\right)$ as the coordinates of the principal points, $\alpha_{j}, \beta_{j}$ the scale factors in image $u$ and $v$ axes, and $\gamma_{j}$ the parameter describing the skewness of the two image axes.

Once the left and camera parameters are estimated, i.e. $\left(\begin{array}{ll}R_{j} & T_{j}\end{array}\right), A_{j}$ and $k_{j 1}, k_{j 2}$, given the image coordinates of any a point in the view we are able to obtain its 3-D coordinates $p=\left[\begin{array}{lll}x & y & z\end{array}\right]^{T}$ in the world coordinate system by triangulation measurement.

\subsection{Spatio-temporal Point Matching}

In our framework, template correlation is realized twice both temporally and spatially. The correlation factors for both cameras are multiplied as weights for the 3-D motion estimation. The computation of correlation is

$$
\begin{gathered}
W_{d x, d y}=\left(\varepsilon \frac{\sum_{h} \sum_{k}\left|I_{h+i 1, k+j 1}^{t-1} \cdot I_{h+i 1+d x, k+j 1+d y}^{t}\right|}{\sqrt{\sum_{h} \sum_{k}\left(I_{h+i 1, k+j 1}^{t-1}\right)^{2}} \cdot \sqrt{\sum_{h} \sum_{k}\left(I_{h+i 1+d x, k+j 1+d y}^{t}\right)^{2}}}+\right. \\
(1-\varepsilon) \frac{\sum_{h} \sum_{k}\left|J_{h+i 2, k+j 2}^{t} \cdot I_{h+i 1+d x, k+j 1+d y}^{t}\right|}{\sqrt{\sum_{h} \sum_{k}\left(J_{h+i 2, k+j 2}^{t}\right)^{2}} \cdot \sqrt{\sum_{h} \sum_{k}\left(I_{h+i 1+d x, k+j 1+d y}^{t}\right)^{2}}},
\end{gathered}
$$

With $I_{i j}^{t-1}, I_{i j}^{t}$ as the intensity value of the pixel $(i, j)$ from the same camera at the time instant $t-1, t$ respectively, $J_{i j}^{t-1}$ as that from the other camera at the time instant $t-1$, $d x, d y$ as estimated displacement values of image points in the horizontal and vertical direction, and $\varepsilon$ is the temporal-spatial matching ratio value (0.6 0.8).

Here a multi-resolution framework is utilized to increase the efficiency with smaller both search region (5x5) and template window (7x7): We construct a quadtree pyramid and start template matching based on the normalized correlation from the coarsest level, the calculated displacement vectors will be propagated to the finer level; The new search region in the current level will be set by this displacement vector, the matching result is from the finest level of the pyramid.

We update the template by an IIR filter:

$$
\Gamma[t]=\mu \Gamma[t-1]+(1-\mu) \Gamma[0],
$$

With $\Gamma[t], \Gamma[0]$ as the current template and the initial template, $\mu$ as the update ratio (0.4 0.6).

\subsection{Robust 3-D tracking}

After the whole motion calculation, the 3-D coordinates would be "adjusted" as follows:

$$
\dddot{p}=R\left(p_{0}+\vec{D}\left(p_{0}\right)\right)+T .
$$

But we must remind, those points as eye corners and nostrils only perform rigid motion, without expression. After this modification, these 3-D points are re-projected into the left and right images as the new search area for the next spatio-temporal template matching, i.e.

$$
s_{j}\left[\begin{array}{c}
P_{j} \\
1
\end{array}\right]=A_{j}\left[\begin{array}{ll}
R_{j} & T_{j}
\end{array}\right]\left[\begin{array}{l}
p \\
1
\end{array}\right],(j=l, r),
$$

With $s_{j}=r_{j 31} x+r_{j 32} y+r_{j 33} z+T_{j z}$.

\section{EXPERIMENT RESULTS}

Our stereo environment is a binocular vision system from the Vision1 Corp. [19]. There are two (Master and Slave) PC computers, and each of them is connected with a Kodak ES310 camera. The synchronization is controlled by the Master computer, the video capture rate is 60 frames/second. Because the color images captured from Vision1 are saved in a special format, we have to transfer this format into a standard format (Bitmap or PPM) first.

Estimated camera intrinsic parameters are given below: 


\section{Left camera}

$\alpha, \gamma, \beta: 1132.29,-21.4687,1128.03$.

$u_{0}, v_{0}: 127.048,92.0548$.

Right camera

$\alpha, \gamma, \beta: 1092.76,-11.5759,1077.38$.

$u_{0}, v_{0}: 175.019,124.301$.

Some stereo tracking results are given in Figure 2. On Pentium 400M, realized by $\mathrm{VC}++$, the processing speed is about 2 frames/second. Here at initialization, we move by mouse our model vertex to fit the first pair of stereo views.

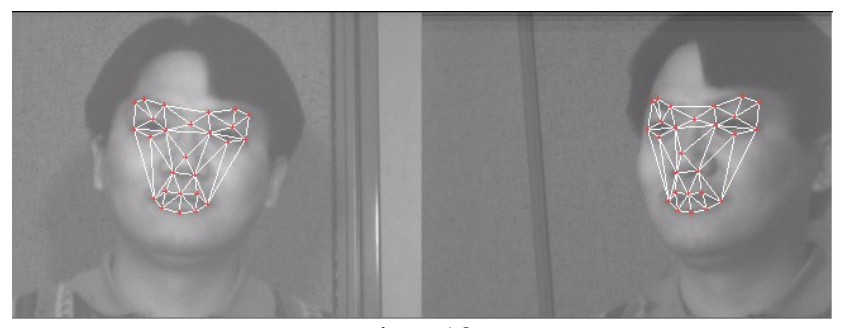

time 10

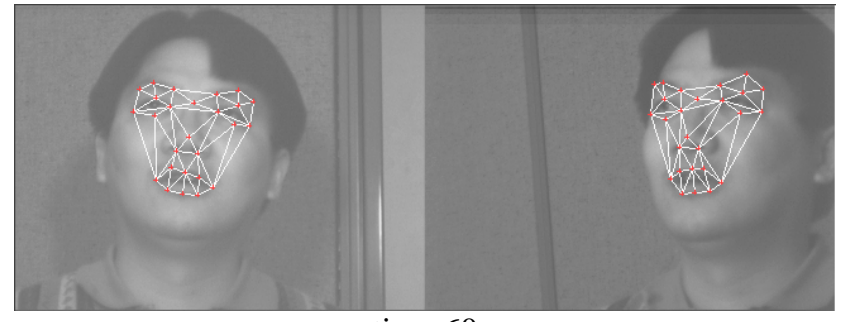

time 60

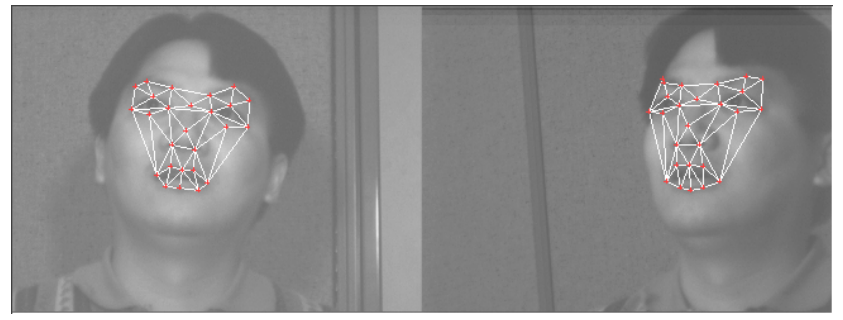

time 110

Figure 2 Stereo facial tracking results

\section{CONCLUSION}

We propose a stereo facial tracking method in which 3$\mathrm{D}$ information of the facial features are fully utilized. We give an efficient representation of the facial motion, and the global motion (head move) and the local motion (expression) can be estimated separately. This tracking result can be applied in facial animation, coding and understanding for $\mathrm{HCI}$.

\section{ACKNOWLEDGEMENT}

This research is supported partially by the NSF Grants CDA96-24396, EIA-99-75019 and IIS-00-85980.

\section{REFERENCES}

[1] Aizawa, K, Huang, T.S. "Model-based image coding advanced video coding techniques for very low bit-rate applications", Proc. of IEEE, 83(2), Feb. 1995.

[2] Arun K, Huang T, Blostein S, "LS fitting of two 3-D point sets", IEEE T-PAMI, 9(5), 1987.

[3] Black M, Yacoob Y, 'Tracking and recognizing rigid and non-rigid facial motions using local parametric models of image motion', ICCV'95, 1995.

[4] Covell, M. "Eigen-points: control-point location using principal component analyses", Int. Conf. on Automatic Face and Gesture Recognition, 1996.

[5] DeCarlo D, Metaxas D, "The integration of optical flow and deformable models with applications to human face shape and motion estimation", CVPR '96.

[6] Edwards G.J., Taylor C.J., Cootes T.F., "Interpreting Face Images using Active Appearance Models", Int. Conf. Face and Gesture Recognition, 1998.

[7] Eisert P et.al, "Model-aided coding: a new approach to incorporate facial animation into motion-compensated video coding”, IEEE T-CASVT, 10(3), pp. 344-358, April 2000.

[8] Gukturk S B et.al, "A data -driven model for monocular face tracking”, ICCV'01, July 2001.

[9] Jebara T, Pentland A, 'Parametrized structure from motion for 3D adaptive feedback tracking of faces', CVPR'97, 1997.

[10] Kumar V, Poggio T, 'Learning-based approach to real time tracking and analysis of faces', Int. Conf. Automatic Face and Gesture Recognition'00, 2000.

[11] Lee M, Ranganath S, "3D deformable face model for pose determination and face synthesis", Int. Conf. Image Analysis and Processing, pp260-265, 1999.

[12] Li Baoxing, Chellapa R, "Gabor Attibutes tracking for face verification”, ICIP'00, pp45-48, 2000.

[13] Matsumoto Y, Zelinsky A, "An algorithm for realtime stereo vision implementation of head pose and gaze direction measurement", IEEE Int. Conf. on Automatic Face and Gesture Recognition, 2000.

[14] Mauer T, Malsburg C, "Tracking and lerarning graphs and pose on image sequences of face", IEEE Int. Conf. on Automatic Face and Gesture Recognition, 1996.

[15] Pighin F. et.al, "Resynthesizing facial animation through 3D model-based tracking". ICCV'99, 1999.

[16] Stroem, J, et.al, "Real-time tracking and modeling of faces: an EKF-based analysis by synthesis approach", Proc. of Workshop on Modeling People, ICCV'99, 1999.

[17] Tao H, Huang T S, 'Explanation-based facial motion tracking using a piecewise Bezier volume deformation model', CVPR'99, 1999.

[18] Tian Yingli et.al, 'Recognizing Upper Face Action Units for Facial Expression Analysis ', CVPR'00, 2000.

[19] Vision1 Stereo System, visit the website http://www.vision1.com.

[20] Zhang Z. "A flexible new technique for camera calibration”. IEEE-PAMI, 22(11), 2000. 